\title{
Measurement of contrast sensitivity function in laser light
}

\author{
Katarzyna SARNOWSKA-HABRAT, BogusŁawa DUBIK, Marek ZAJĄC
}

Institute of Physics, Wrocław University of Technology,

Wybrzeże Wyspiańskiego 27, 50-370 Wrocław, Poland

\begin{abstract}
Visual quality depends on many factors of different nature and therefore it is not easy to define it. Different measures are used to describe vision quality, such as: two-point resolution, visual acuity, contrast sensitivity function (CSF), etc. We concentrate especially on CSF. There are two important factors affecting the character of CSF. One of them is connected with the optical transfer function (OTF) of the eye and the second one with the retinal response. Typically, CSF is measured in incoherent light. Due to the dependence of CSF on both mentioned above factors simultaneously it is impossible to extract the information on the eye optical system only. Typically, CSF is measured in spatially and temporarily incoherent light. We hope that additional information offered by CSF measurement in quasi-monochromatic and spatially partially coherent light can help to solve this problem.
\end{abstract}

Keywords: vision quality, contrast sensitivity function, laser light.

\section{Introduction}

Estimation of imaging quality is one of the most important problems in optics of vision. The crux of the matter lies in the fact that vision quality depends on so many factors. They are, among others: quality of image formed by the optical system of an eye (retinal image), detection of this image by the retina, preprocessing of the neural signals and their transmission from the retina to the brain, and finally, the interpretation of these signals by visual cortex [1]-[3].

The quality of retinal image can be described using such parameters, defined in physical optics, as point spread function, aberrations or optical transfer function (OTF). Well known models explain resolution limits as well as contrast degradation in the image of a periodic test of high spatial frequency. These models do not include, however, the influence of all factors affecting vision. At least one more has to be taken into account. We mean preprocessing of impulses produced by individual cones by retinal neurons [4], [5]. The magnitude of a signal generated by ganglion cells depends on the comparison of impulses originating from retinal excitation field and exhibition field performed by horizontal cells (Fig. 1). 


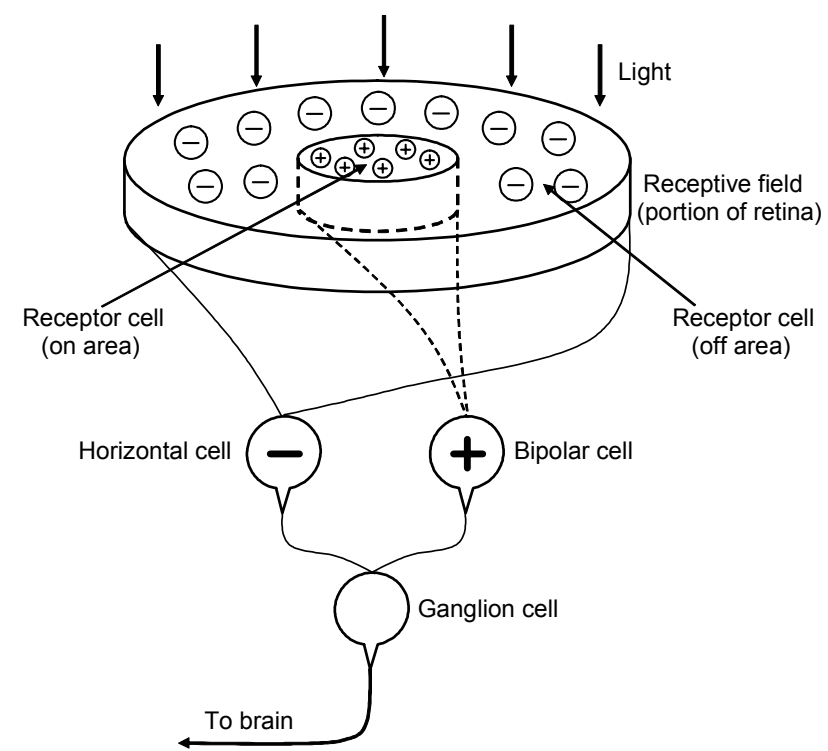

Fig. 1. Detection of light in the retina and preprocessing of neural signals (adapted from [3]).

If the light intensity distribution on the retina has a form of periodic fringes, then the retinal response depends on spatial frequency and contrast of these fringes. If spatial frequency corresponds to the typical size of excitation field its magnitude is the greatest. If fringes frequency is substantially lower or higher, the signal sent to the brain is less intensive. This model explains relatively low sensitivity of the eye for periodic objects of low spatial frequency (Fig. 2).

Both mechanisms mentioned above: contrast degradation in retinal image of high frequency periodic objects, and dependence of retinal sensitivity on spatial frequency of such objects influence contrast sensitivity function (CSF). This function is defined
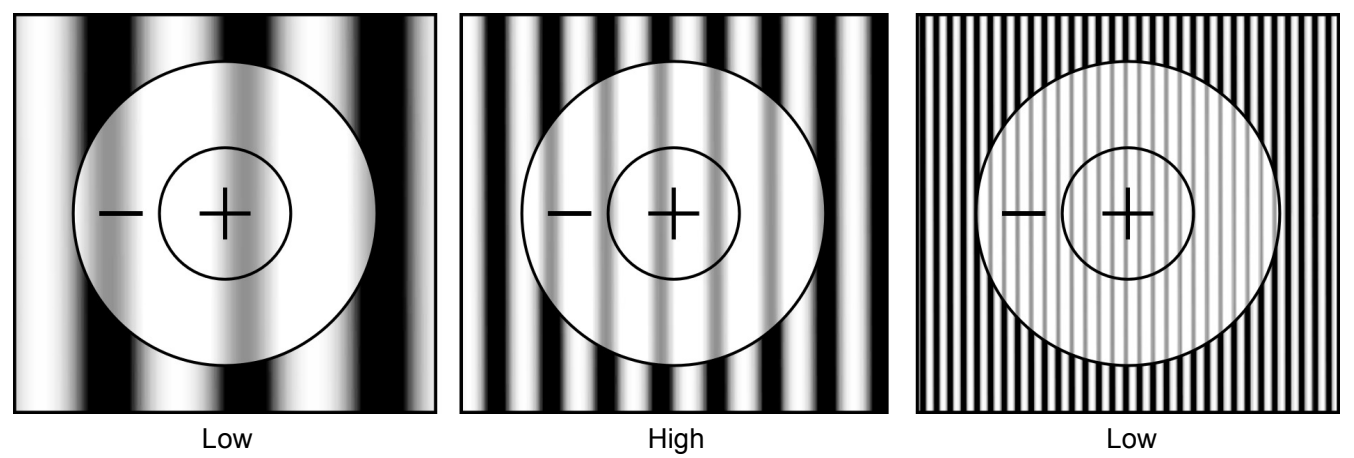

Fig. 2. Retinal response. 


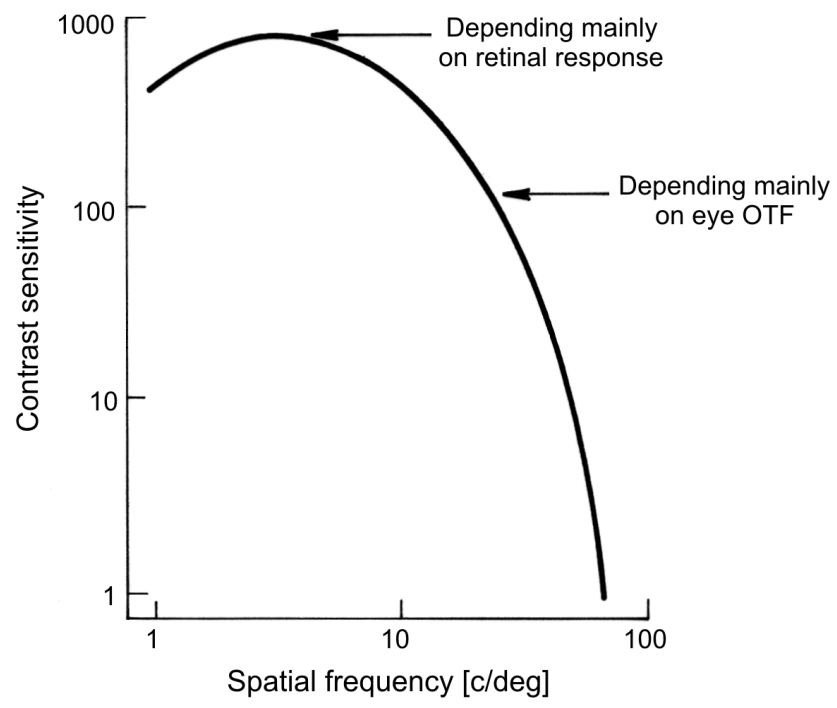

Fig. 3. Typical shape of CSF.

as the dependence of the lowest recognized contrast of periodic test-object presented to the investigated person on the spatial frequency of an object. Typical shape of CSF is presented in Fig. 3. For spatial frequencies equal to 3 to 5 lines per degree the eye sensitivity is the highest. It falls down for lower spatial frequency due to lower sensitivity of retina and for spatial frequencies due to blurring of retinal image.

\section{Measurement}

The procedure of CSF measurement is based on presenting periodic tests of different spatial frequencies and contrast to the tested person and then asking whether he/she can recognize the test structure (orientation of fringes). The simple test plates printed on white paper are typically used [6]. Because of a limited number of individual test patterns, the accuracy of such means plays a crucial role [7], [8]. In this case the limited number of gray levels and size of particular pixels also limits the measurement accuracy (see Fig. 4).

As a better solution the use of fringe field obtained in any interferometric device as a test object can be proposed. In this paper a Mach-Zehnder interferometer for generating light field of cosinusoidal modulation is used (Fig. 5). Fringe spacing and direction were changed by tilting one of the mirrors and fringe contrast by rotating a polarizer inserted in one of the interferometer's arms. As light source lasers giving light of wavelength $\lambda_{1}=633 \mathrm{~nm}$ and $\lambda_{2}=532 \mathrm{~nm}$ were used.

The investigated person was seated before the interferometer and was asked to observe the fringes from the distance of about $35 \mathrm{~cm}$ (for near vision) - see Fig. 6 . The head was fixed by a chinrest and one eye was covered. The measurement strategy 


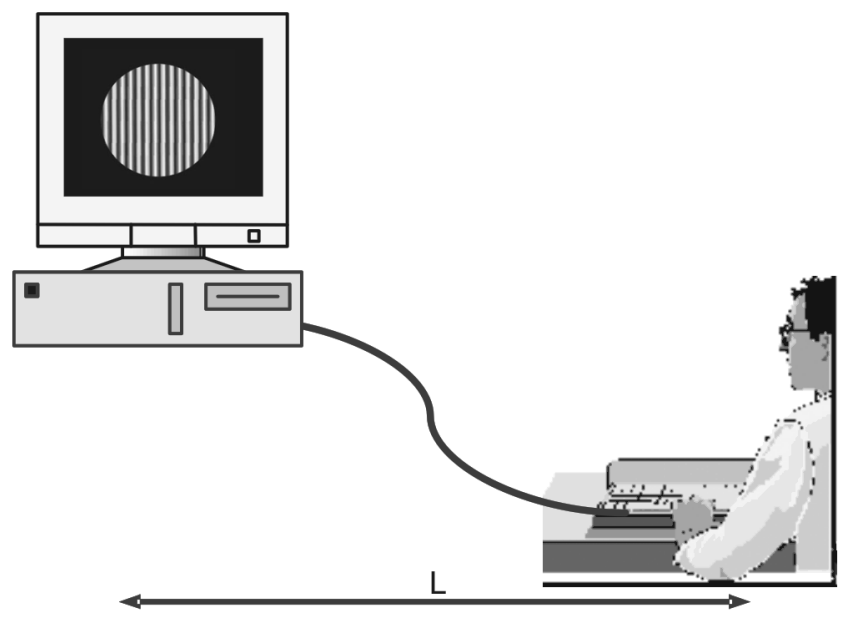

Fig. 4. Conventional setup for CSF measurement.

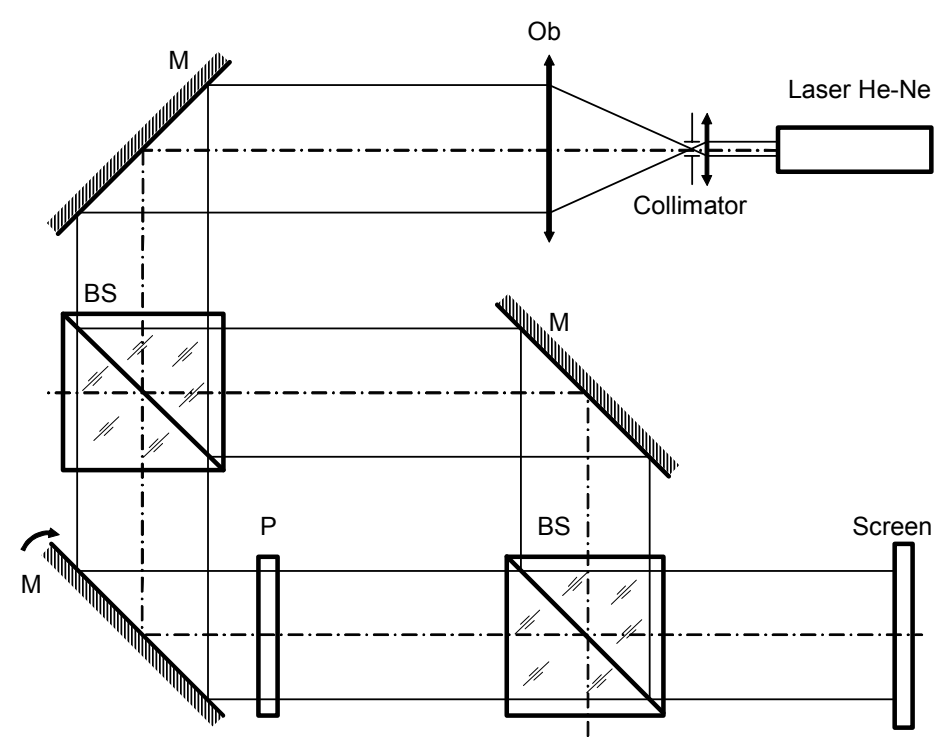

Fig. 5. Interferometer for generating fringed pattern $(\mathrm{Ob}$ - objective, $\mathrm{M}-$ mirror , $\mathrm{P}$ - polarizer, $\mathrm{BS}-$ beam splitter).

assumed a slow increase of the fringe contrast until the investigated person could recognize the test pattern (its orientation). To avoid errors caused by "learning" both: the fringes direction and spacing were changed at random.

The Mach-Zehnder interferometer generates non-localized fringe field. Therefore it is difficult to accommodate the eye on a desired distance. In order to make eye fixation possible the interference fringes are imaged onto diffusing screen. This fact 


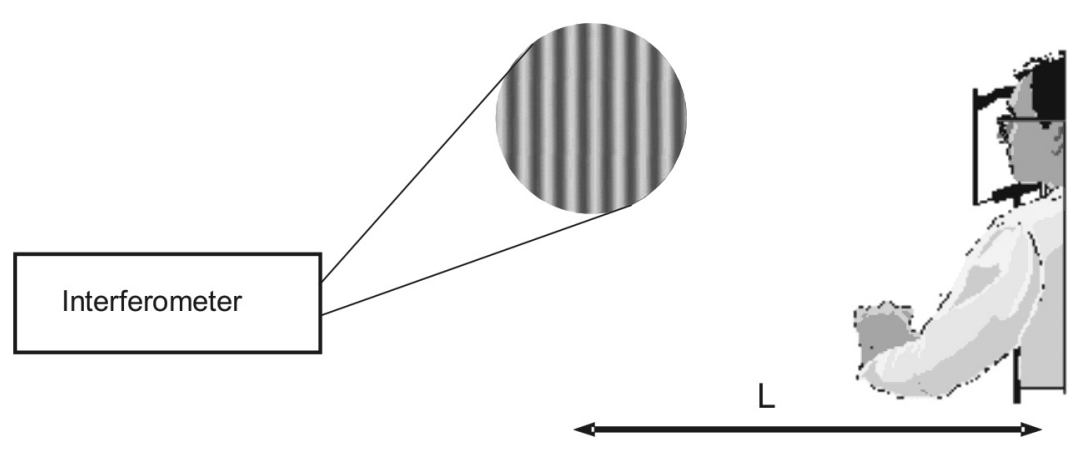

Fig. 6. Interferometric setup for CSF measurement.

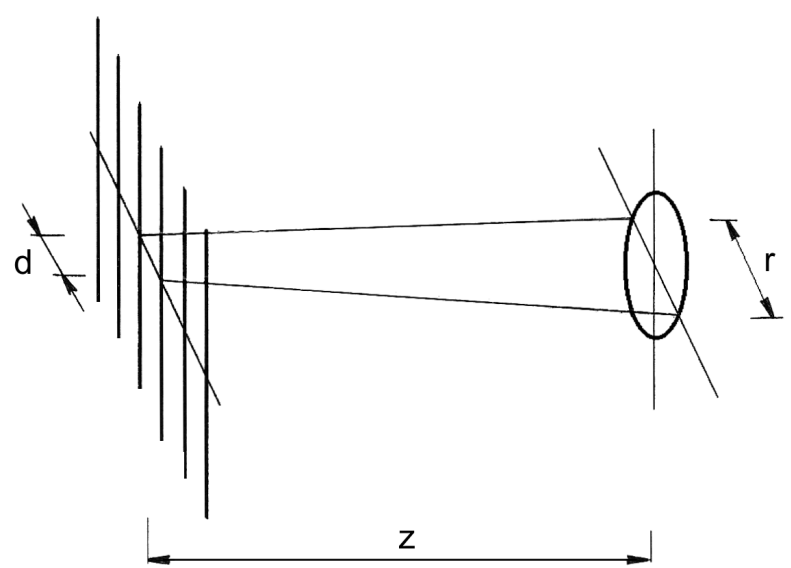

Fig. 7. Evaluation of the coherence state $(d$-width of an individual bright strip, $z$ - observation distance, $r$-diameter of the eye pupil).

has a very important consequence. The interferometer itself operates in temporarily and spatially coherent light and generates high contrast interference fringes on a screen. Thanks to its microstructure the screen acts as a random phase diffuser and introduces additional phase shift to particular points of the light field which form fringe structure. By rotating the screen we introduce quasi-random changes in the phase of light emitted from it. Therefore each single strip of the periodic structure of interference field on the screen can be treated as a spatially incoherent secondary light source. The state of coherence changes when the light propagates from the diffuser towards the eye of an investigated person. In the plane where a pupil of the eye is located (see Fig. 7) the degree of partial coherence $\gamma$ can be estimated from the following formula [9]

$$
|\gamma|=\frac{2 J_{1}\left(\frac{2 \pi r d}{2 \lambda z}\right)}{\frac{2 \pi r d}{2 \lambda z}}
$$


where $d$ is the width of a secondary light source, $z$ - the distance from it to the analysed plane, $r$ - the distance between a pair of typical points in this plane, $J_{1}-$ Bessel function of first order, first kind. To estimate the value of $\gamma$ let us assume the following numerical data. The periodic structure has a spatial frequency 30 line pairs/angular degree, so the width of an individual bright strip $d=1 \mathrm{~mm}$. Observation distance $z=350 \mathrm{~mm}$, diameter of the eye pupil $r=4 \mathrm{~mm}$, light wavelength $\lambda=0.0006 \mathrm{~mm}$. The degree of coherence for pairs of points lying within the eye pupil is not less than $|\gamma|=0.1$.

From this calculation it follows that we cannot describe the imaging process in an optical system of the eye in terms of neither completely coherent light nor completely incoherent light. Rather partial coherence of varying degree has to be assumed.

Due to high coherence of light in the interferometer the speckles appear on the screen [9] making the recognition of the fringe structure difficult. However, thanks to the rotation of a ground glass screen the speckle structure disappears.

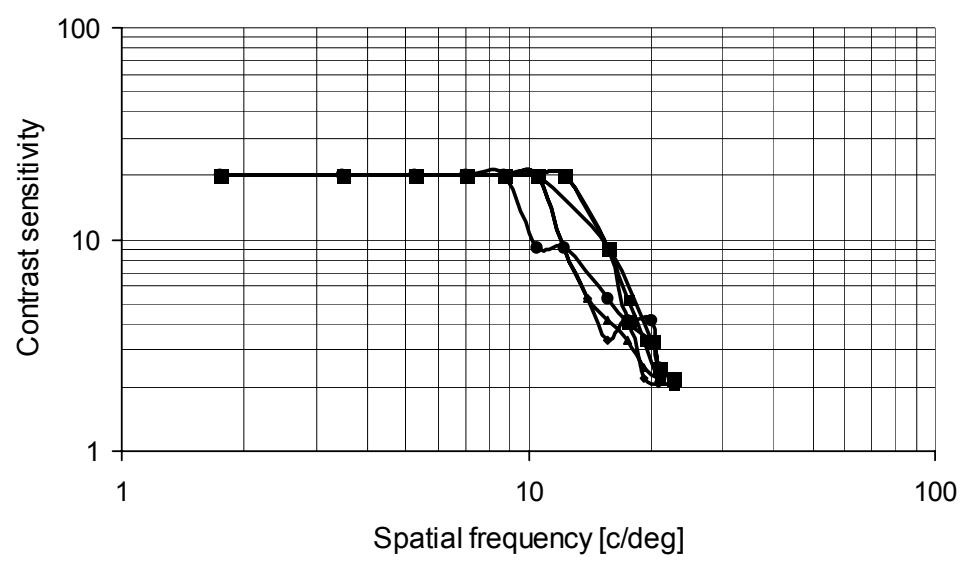

a

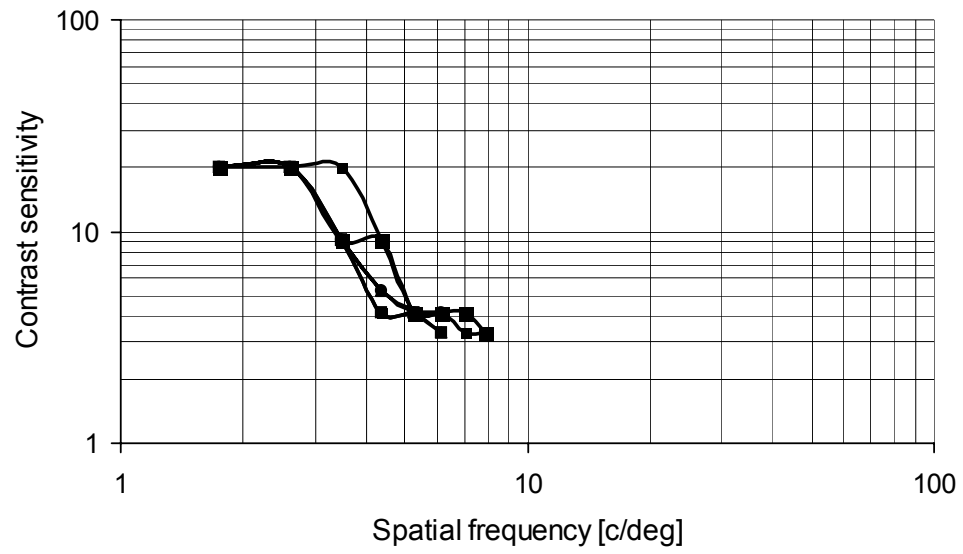

b

Fig. 8. CSF measured in 7 consecutive days for two persons (figures $\mathbf{a}$ and $\mathbf{b}$, respectively). 


\section{Results of measurement}

The described method of CSF measurement is typically a subjective one. Therefore, an influence of the examined person's state on the measurement process cannot be neglected. A number of human factors such as health condition, fatigue, intellectual ability etc., can influence the result of measurements. In order to estimate the importance of these human factors we performed a series of measurements of CSF for two persons in seven consecutive days. The results are shown in Fig. 8a, b. As it can be seen from the data presented therein the scattering of the results is not serious.

The data presented in Fig. 8a, b were obtained in the measurements in which a ground glass serving as an observing screen was not moving. A speckling effect was present. By rotating the glass the speckling effect can be eliminated [10]. As it follows from our experiments the shape of CSF if measured with the speckling effect present was different than the respective CSF if measured with the speckling effect eliminated by rotating the screen. Typical shapes of CSF curves for the same person and speckles present or absent are presented in Fig. 9.

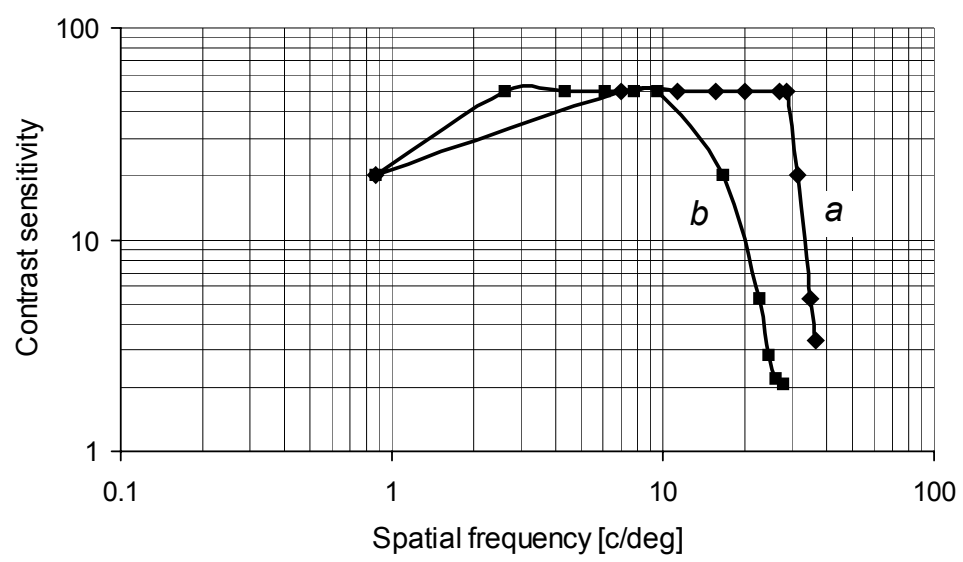

Fig. 9. CSF as measured: with speckles $(a)$, without speckles $(b)$.

In our investigations we concentrated on the CSF functions measured for young persons (20-25 years of age) with no refraction error or with refraction error corrected. Typical results for such group of subjects were similar - almost constant value of CSF and then rapid drop down near the limit frequency. It is illustrated in several examples presented in Fig. 10a-d. Such shape of CSF is not similar to the typical one illustrated schematically in Fig. 3. The reason of this discrepancy is probably caused by the fact that the imaging conditions of high spatial frequency tests are nearer to coherent illumination than to pure incoherent illumination as it is in typical CSF measurements. Optical transfer function of the eye optical system is more close to coherent than incoherent one. 

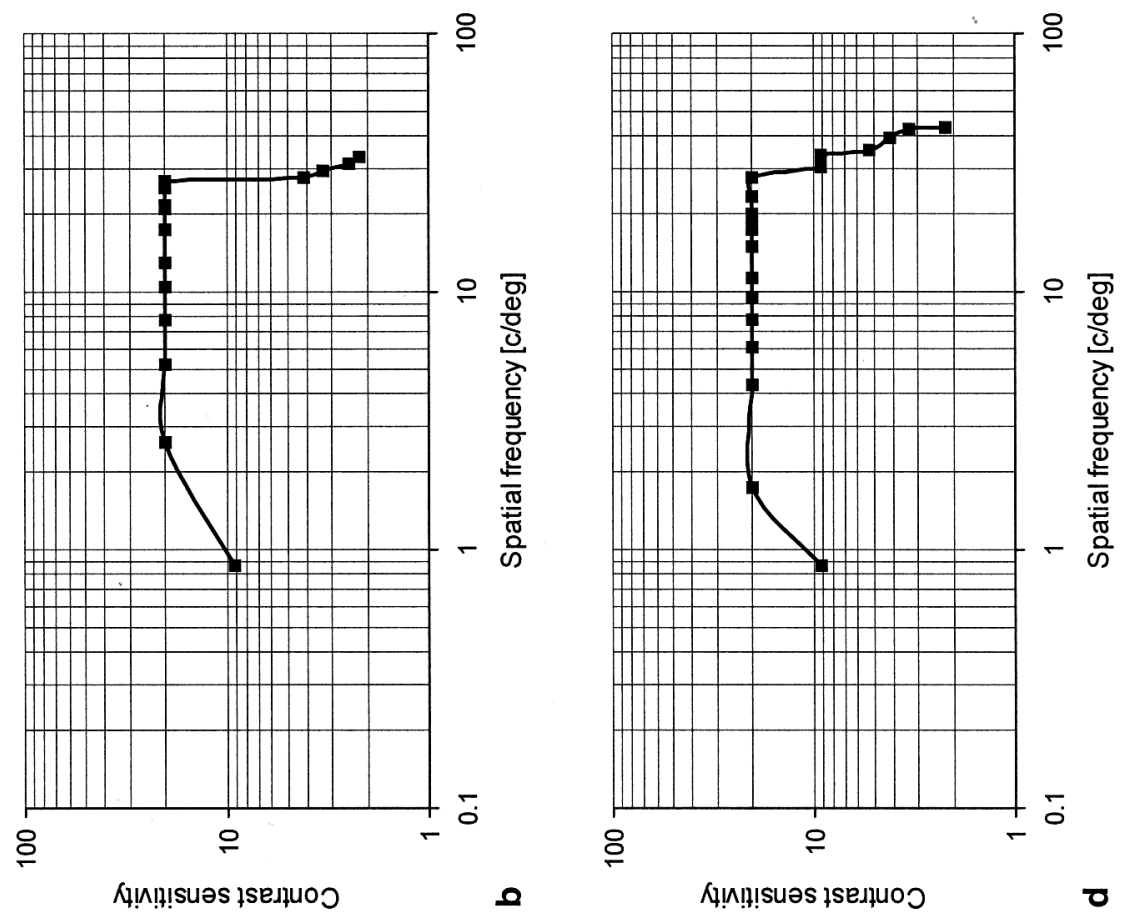

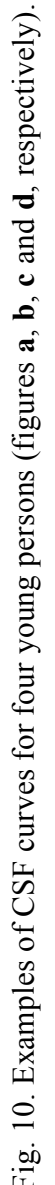
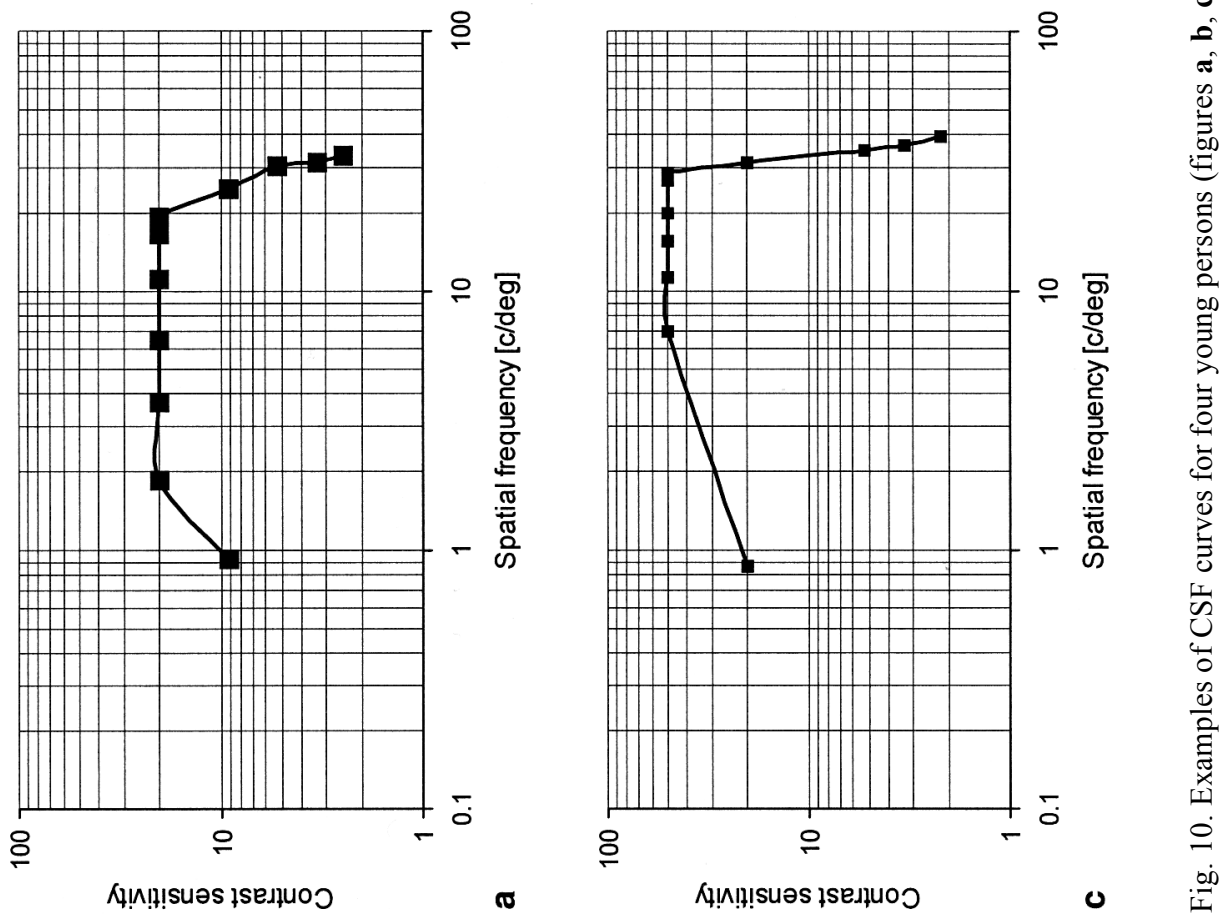


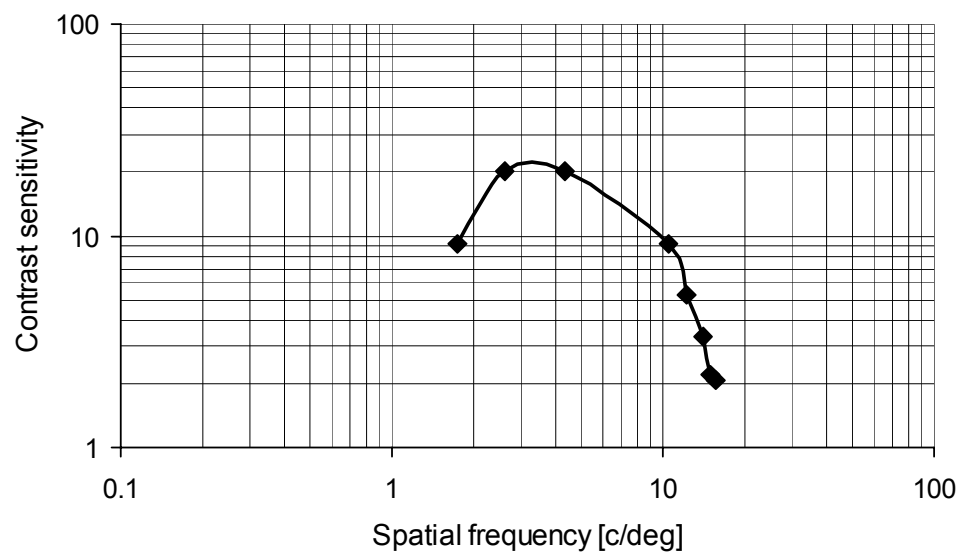

a

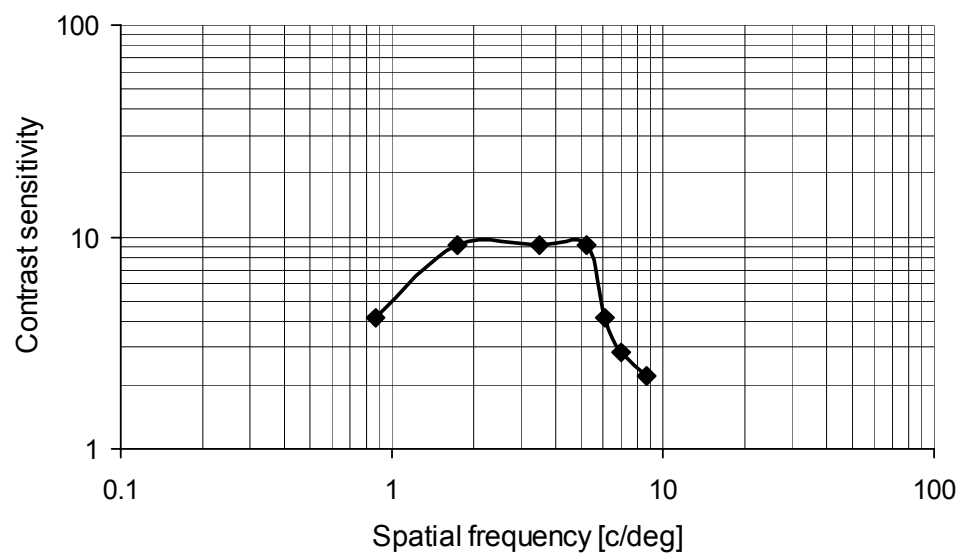

Fig. 11. Examples of CSF functions measured for older persons.

The situation is different when we measure CSF of older persons, especially with substantial refraction errors. Typical CSF curves measured for persons of 30-50 years of age, with noticeable presbyopia, are shown in Fig. 11. High spatial frequancy part of CSF function is evidently altered, probably by an uncompensated refraction error.

\section{Conclusions}

We feel that CSF as measured in laser light is more sensitive to eye aberrations such as those caused by a non-precise correction of refraction errors than the respective CSF as measured in typical incoherent illumination. We think also that by the comparison of the shapes of CSF measured in incoherent and coherent illuminations it will be possible to estimate the influence of an optical system of the eye and retinal response on the vision quality separately. 
Acknowledgments - This paper was presented at XIV Polish-Czech-Slovak Optical Conference Wave and Quantum Aspects of Contemporary Optics, Krzyżowa, Poland, 14-17 September 2002. The authors thank prof. Jan Jabczyński for valuable comments and remarks.

\section{References}

[1] Ginsburg A.P., Am. J. Optom. 61 (1984), 403.

[2] Corvin T.R., Richman J.E., Am. J. Optom. 63 (1986), 413.

[3] Sekiguchi M., Williams D.R., Brainord D.H., J. Opt. Soc. Am. A 10 (1993), 2105.

[4] Lindsay P.H., Norman D.A., Procesy przetwarzania informacji u człowieka (in Polish), [Ed.] PWN, Warszawa 1984.

[5] Kaiser P.K., Boyton R.M., Human Color Vision, Opt. Soc. Am., Washington 1996.

[6] KęDzia B., Klinika Oczna, No. 90 (1988), 178 (in Polish).

[7] KęDzia B., Niworowski M., Optyka - Optometria, No. 6 (1993), 8 (in Polish).

[8] Kaziath Latham, Ophthal. Physiol. Opt. 18 (1998), 2.

[9] Beran M.J., Parrent, G.B. Theory of Partial Coherence, Prentice-Hall, Englewood Cliffs, 1964.

[10] Hrabovsky M., Basa Z., Horvath P., Koherencni zrnistost v optice (in Czech), Universita Palackeho, Olomouc 2001. 